\title{
Uso do pó de rocha basáltica como fertilizante alternativo na cultura da soja
}

\author{
Use of basalt rock powder as an alternative fertilizer culture of soybean \\ Uso de polvo de roca basáltica como fertilizante alternativo en el cultivo de soja
}

Luciene Kazue Tokura

ORCID: https://orcid.org/0000-0001-9758-0141

Universidade Estadual do Oeste do Paraná, Brasil

E-mail: lucienetokura@gmail.com

Cleidimar João Cassol

ORCID: https://orcid.org/0000-0002-2433-7084

Universidade Federal da Grande Dourados, Brasil

E-mail: cleidimar_cassol@hotmail.com

Robervaldo Soares da Silva

ORCID: https://orcid.org/0000-0002-0214-4820

Universidade Federal da Grande Dourados, Brasil

E-mail: robervaldo.soares@yahoo.com.br

Willian Isao Tokura

ORCID: https://orcid.org/0000-0001-9363-793X

Universidade Federal de Goiás, Brasil

E-mail: willianisaotokura@hotmail.com

Adama Gning

ORCID: https://orcid.org/0000-0003-2231-1961

Universidade Federal da Grande Dourados, Brasil

E-mail: gningadama83@gmail.com

Priscila Marques Kai

ORCID: https://orcid.org/0000-0002-4767-3718

Universidade Federal da Grande Dourados, Brasil

E-mail: priscila.kai@hotmail.com

\section{Resumo}

Objetivou-se com o presente estudo avaliar o efeito residual da adição de pó de rocha basáltica associado ou não a adubação química, sobre os atributos químicos do solo e produtividade da cultura da soja. O delineamento experimental foi de blocos casualizados em esquema fatorial $(5 \times 2)$, testando, cinco doses de pó de rocha $(0 ; 2,5 ; 5,0$; 7,5 e 10,0 $\mathrm{Mg} \mathrm{ha}^{-1}$ ), com ou sem adubação química de NPK na formulação 05-25-06, com quatro repetições. O experimento foi conduzido em condições de campo. Foram avaliadas as características agronômicas de altura final de 
plantas, altura da inserção do primeiro legume, diâmetro do coleto, peso de 1000 grãos, produtividade da soja e os atributos químicos do solo. A adubação química influenciou os teores dos nutrientes $\mathrm{P}, \mathrm{K}$ e Mn no solo. Na camada de 0-10 cm houve aumento dos teores de P e K. Na camada de 10-20 cm ocasionou redução no teor de Mn. O residual da aplicação das doses de pó de basalto ocasionou a redução das concentrações foliares de $\mathrm{P}, \mathrm{Cu}, \mathrm{Zn}$. A adubação química complementar favoreceu a redução da concentração do $\mathrm{Mg}$ foliar. Altura de plantas, diâmetro do coleto, peso de grãos e produtividade foram maiores nos tratamentos que receberam a adubação química. A pequena liberação dos nutrientes do pó de basalto indica que tal material não pode ser utilizado como a principal fonte de nutrientes às plantas.

Palavras-chave: Basalto; Glycine max; Adubação.

\begin{abstract}
The objective of this study was to evaluate the residual effect of adding basaltic rock powder associated or not with chemical fertilization on the chemical attributes of the soil and the productivity of the soybean crop. The experimental design was randomized blocks in a factorial scheme $(5 \mathrm{x} 2)$, testing five doses of rock dust $(0 ; 2.5 ; 5.0 ; 7.5 \mathrm{and} 10.0 \mathrm{Mg}$ ha-1), with or without fertilization. NPK chemistry in formulation 05-25-06, with four replications. The experiment was carried out under field conditions. The agronomic characteristics of the final height of plants, the height of the insertion of the first vegetable, the diameter of the collection, the weight of 1000 grains, soybean products, and the chemical attributes of the soil were evaluated. Chemical fertilization influenced the levels of nutrients $\mathrm{P}, \mathrm{K}$, and $\mathrm{Mn}$ in the soil. In the 0-10 cm layer, there was an increase in the levels of P and K. In the 10-20 cm layer; the Mn content was reduced. The residual application of the doses of basalt powder caused the reduction of leaf concentrations of $P$, $\mathrm{Cu}, \mathrm{Zn}$. The complementary chemical fertilization favored the reduction of leaf $\mathrm{Mg}$ concentration. Plant height, stem diameter, grain weight, and productivity were higher in treatments that received chemical fertilization. The minor release of nutrients from the basalt powder indicates that such material cannot be used as the primary source of nutrients for plants.
\end{abstract}

Keywords: Basalt; Glycine max; Fertilization.

\title{
Resumen
}

El objetivo de este estudio fue evaluar el efecto residual de la adición de polvo de roca basáltica asociado o no a la fertilización química, sobre los atributos químicos del suelo y la productividad del cultivo de soja. El diseño experimental fue de bloques al azar en un esquema factorial $(5 \times 2)$, probando cinco dosis de polvo de roca $(0 ; 2.5 ; 5.0$; 7.5 y 10.0 Mg ha-1), con o sin fertilización. Química NPK en formulación 05-25-06, con cuatro repeticiones. El experimento se llevó a cabo en condiciones de campo. Se evaluaron las características agronómicas de altura final de plantas, altura de inserción de la primera leguminosa, diámetro de la colección, peso de 1000 granos, productividad de soja y atributos químicos del suelo. La fertilización química influyó en los niveles de nutrientes P, K y Mn en el suelo. En la capa de 0-10 cm hubo un aumento de los niveles de P y K. En la capa de 10-20 cm se produjo una reducción del contenido de Mn. La aplicación residual de las dosis de polvo de basalto provocó la reducción de las concentraciones foliares de $\mathrm{P}, \mathrm{Cu}, \mathrm{Zn}$. La fertilización química complementaria favoreció la reducción de la concentración de $\mathrm{Mg}$ foliar. La altura de la planta, el diámetro del tallo, el peso del grano y la productividad fueron mayores en los tratamientos que recibieron fertilización química. La pequeña liberación de nutrientes del polvo de basalto indica que dicho material no puede usarse como la principal fuente de nutrientes para las plantas.

Palabras clave: Basalto; Glycine max; Fertilizantes.

\section{Introdução}

O Brasil é um dos países que mais produz soja no mundo, com produção recorde estimada em 120,9 milhões de toneladas, ganho de 5,1\% em relação à safra 2018/19. Os grãos de soja se encontram na liderança do ranking de exportações, alcançando a marca de mais de 80 milhões de toneladas de grãos de soja (Conab, 2020). Entretanto, considerando toda a cadeia agrícola nacional e a baixa fertilidade natural dos solos brasileiros, o país tornou-se grande importador de fertilizantes. Em 2018, o Brasil foi o país que mais importou fertilizantes nitrogenados, fosfatados e potássicos no mundo, sendo gasto 2,68 bilhões, 360,57 milhões e 3,35 bilhões de dólares, respectivamente com a importação dos fertilizantes (Tridge, 2019a, b e c).

Segundo Polidoro (2017), 79\% do total de fertilizantes consumidos no país são importados, o que torna a agricultura brasileira muito dependente do mercado externo e do preço do dólar. Para diminuir essa dependência, que pesa sobre produtores e sobre a balança comercial do país, a pesquisa agrícola nacional está desenvolvendo e incentivando o uso de fontes alternativas de nutrientes. 
Dessa forma, a utilização de pó de rochas ou rochagem apesar de não ser um conceito novo tem sido objeto de pesquisas oficiais e de portfólios de empresas, principalmente depois que o Ministério de Agricultura, Pecuária e Abastecimento (MAPA), através da Lei 12.890 de 10 de dezembro de 2013 e a Instrução Normativa ${ }^{\circ}$ 5, de 10 de março de 2016, regulamentaram a produção, registro e comércio do pó de rocha na agricultura, atualmente chamado de "Remineralizadores", sendo o material de origem mineral que tenha passado apenas por redução e classificação de tamanho por processos mecânicos e que altere os índices de fertilidade do solo, por meio da adição de macro e micronutrientes para as plantas, bem como promova a melhoria das propriedades físicas, físico-químicas e/ou da atividade biológica do solo, além das quantidades máximas permitidas de contaminantes como o Arsênio (As), Cádmio (Cd), Mercúrio ( $\mathrm{Hg}$ ) e Chumbo $(\mathrm{Pb})$, além de servir como parâmetro comparativo na prospecção de novos recursos (Brasil, 2016).

A utilização de remineralizadores tem potencial para reduzir a dependência de insumos externos e melhorar a eficiência na manutenção da fertilidade do solo através da lenta e gradual diminuição do uso de fertilizantes industriais, em manejos que permitam o incremento de matéria orgânica no solo e promovam condições favoráveis para a sobrevivência e estabelecimento de microrganismos do solo em geral, que através de ácidos orgânicos, contribuem para a solubilidade de nutrientes e, em especial, os fungos micorrízicos arbusculares (FMAs), que podem acelerar o processo de intemperismo, desde que estejam em contato direto com o pó de (rocha) rochagem, assim, possivelmente se beneficiar de um maior efeito residual e liberação gradativa de nutrientes para a solução do solo (Edward, et al., 2016).

Klein (2020) testou doses crescentes de pó de basalto em dois Latossolos Distroférricos Vermelhos sendo um de textura argilosa e outro de textura média e constatou que houve melhora significativa nos atributos químicos do solo de textura média, enquanto que, o solo de textura argilosa por apresentar maior fertilidade natural não apresentou respostas significativas para a maioria dos atributos químicos do solo.

Segundo Schmidt et al. (2019) a substituição total de fertilizantes químicos solúveis pelo pó de rocha não alterou os atributos químicos do solo, mas os autores relataram efeito positivo nos componentes de produção, onde foi constatado maior número de vagens com três grãos, nos tratamentos que receberam $2 \mathrm{Mg} \mathrm{ha}^{-1}$ de um blend de granito, mármore dolomítico e basalto, quando comparado com a soja do tratamento sem adubação.

Segundo Alovisi et al. (2020), o pó de basalto pode ser considerado como uma fonte alternativa de fertilizante e corretivo do solo de baixo custo, entretanto, a baixa solubilidade do pó de basalto indica que tal material não pode ser utilizado como a principal fonte de nutrientes às plantas.

Assim, objetivou-se com o trabalho avaliar o efeito residual da aplicação do pó de rocha basáltica, associado ou não com adubação química adicional, sobre os atributos químicos do solo e na produtividade da soja.

\section{Metodologia}

\subsection{Descrição do local, clima e solo}

O experimento foi conduzido durante o ano agrícola 2018/2019, em condição de campo, na Fazenda Experimental de Ciências Agrárias da Universidade Federal da Grande Dourados (UFGD), no município de Dourados, MS, localizado a 434m de altitude, $54^{\circ} 59^{\prime} 13^{\prime \prime}$ de longitude Oeste e de $22^{\circ} 14^{\prime} 08^{\prime \prime}$ de latitude Sul.

O clima de Dourados foi classificado como Cwa, clima úmido e inverno seco, as médias anuais de temperatura são de $22^{\circ} \mathrm{C}$ e a precipitação varia entre $500 \mathrm{~mm}$ e $1500 \mathrm{~mm}$ anuais (Fietz, et al., 2017).

No início do experimento, foram aplicadas a lanço sem incorporação cinco doses de pó de basalto $(0 ; 2,5 ; 5,0 ; 7,5$ e $10 \mathrm{Mg} \mathrm{ha}^{-1}$ ). Cultivou-se na área a cultura da soja (safra 2017/18) e milho (safra 2018). Posteriormente, no terceiro cultivo, na safra 2018/2019 cultivou-se a soja para verificar a resposta ao efeito residual do pó de rocha aplicado antes da safra 2017/2018. 
O pó de rocha aplicado ao solo é uma rocha basáltica. Foi utilizado material residual fino resultante da operação de britagem. A granulometria do pó de basalto aplicado no experimento está em acordo com as normas da ABNT para classificação de corretivos e fertilizantes, sendo que $100 \%$ do produto passou em peneira de malha $0,84 \mathrm{~mm}$ e $50 \%$ em peneira de malha 0,3 mm. O valor de $\mathrm{pH}$ (suspensão 1:1) do material é de 9,7 e a composição química total da rocha basáltica é de:

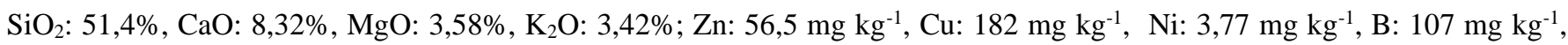
Cl: $798 \mathrm{mg} \mathrm{kg}^{-1}$, Co: 9,65 mg kg${ }^{-1}$, Fe: $22.000 \mathrm{mg} \mathrm{kg}^{-1}$, Mn: $394 \mathrm{mg} \mathrm{kg}^{-1}$, os elementos Cd, As, $\mathrm{Pb}, \mathrm{Hg}$ e Mo se encontravam abaixo da faixa de quantificação. Os elementos macro e traço foram determinados por espectrometria de emissão, ICP.

O solo da área foi classificado como LATOSSOLO VERMELHO Distroférrico, de textura argilosa (Santos et al., 2013), apresentando características químicas (Tabela 1), avaliadas de acordo com metodologia de Claessen (1997).

Tabela 1: Atributos químicos do solo, determinadas em amostras de solo coletadas nas camadas $0-10 \mathrm{~cm}$ e $10-20 \mathrm{~cm}$ antes da implantação do experimento. Dourados - MS, 2019.

\begin{tabular}{|c|c|c|c|c|c|c|c|c|c|c|c|c|}
\hline \multirow{2}{*}{$\begin{array}{c}\text { Profundidade } \\
\text { de coleta }\end{array}$} & \multicolumn{2}{|c|}{$\mathrm{pH}$} & \multirow{2}{*}{$\begin{array}{l}\text { M.O } \\
\mathrm{g} \mathrm{dm}^{-3}\end{array}$} & \multirow{2}{*}{ 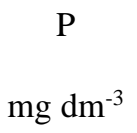 } & K & $\mathrm{Ca}$ & $\mathrm{Mg}$ & $\mathrm{H}+\mathrm{Al}$ & $\mathrm{Al}$ & SB & CTC & \multirow{2}{*}{$\begin{array}{l}\mathrm{V} \\
\%\end{array}$} \\
\hline & $\mathrm{H}_{2} \mathrm{O}$ & $\mathrm{CaCl}_{2}$ & & & \multicolumn{7}{|c|}{ 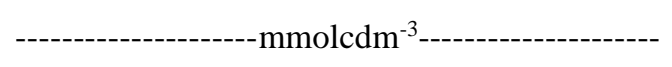 } & \\
\hline$(0-10 \mathrm{~cm})$ & 6,0 & 5,4 & 30 & 13 & 5,1 & 47 & 23 & 43 & 0 & 75 & 118 & 63 \\
\hline$(10-20 \mathrm{~cm})$ & 5,5 & 4,7 & 22 & 6 & 2,3 & 29 & 14 & 55 & 3 & 45 & 101 & 45 \\
\hline
\end{tabular}

Fonte: Autores.

\subsection{Delineamento experimental e tratamentos}

O delineamento experimental foi em blocos casualizados, em esquema fatorial 5x2, utilizando-se de 4 repetições. Os tratamentos foram constituídos de cinco doses de pó de rocha basáltica $\left(0 ; 2,5 ; 5,0 ; 7,5\right.$ e $\left.10 \mathrm{Mg} \mathrm{ha}^{-1}\right)$ com e sem adubação química adicional (05-25-06 NPK $200 \mathrm{~kg} \mathrm{ha}^{-1}$ ). Os tratamentos foram identificados como: T1 (controle); T2 (sem adição do pó de rocha + adubação química adicional); T3 (2,5 $\mathrm{Mg} \mathrm{ha}^{-1}$ de pó de rocha e sem adubação química adicional); T4 (2,5 Mg ha-1 de pó de rocha e com adubação química adicional); T5 (5,0 $\mathrm{Mg} \mathrm{ha}^{-1}$ de pó de rocha e sem adubação química adicional); T6 (5,0 $\mathrm{Mg} \mathrm{ha}^{-1}$ de pó de rocha e com adubação química adicional); $\mathrm{T} 7$ (7,5 $\mathrm{Mg} \mathrm{ha}^{-1}$ de pó de rocha e sem adubação química adicional); T8 (7,5 $\mathrm{Mg} \mathrm{ha}^{-1}$ de pó de rocha e com adubação química adicional); T9 (10 Mg ha ${ }^{-1}$ de pó de rocha e sem adubação química adicional); T10 (10 $\mathrm{Mg} \mathrm{ha}^{-1}$ de pó de rocha e com adubação química adicional.

\subsection{Condução do experimento}

A semeadura da soja, variedade Monsoy 6410, foi realizada no dia 14 de novembro de 2018, com semeadora adubadora, modelo Semeato, equipada com sete linhas, na densidade de semeadura de 16 plantas $\mathrm{m}^{-1}$. A parcela foi constituída por sete linhas espaçadas entre si de $0,45 \mathrm{~m}(3,15 \mathrm{~m})$, com $5 \mathrm{~m}$ de comprimento, com área total da parcela 15,75 $\mathrm{m}^{2}$. Nas parcelas em que os tratamentos constituíam de adubação química complementar foi adicionado a dose de $200 \mathrm{~kg} \mathrm{ha}^{-1} \mathrm{da}$ fórmula 05-25-06 (NPK).

O controle de plantas daninhas foi realizado com o herbicida glifosato, na dose de três litros por hectare. O controle de percevejos foi feito com o inseticida Tiametoxam + Lambda-Cialotrina na dose de $200 \mathrm{~mL} \mathrm{ha}^{-1}$. 


\subsection{Variáveis analisadas}

Foram avaliadas a altura da planta (ALT), altura de inserção do primeiro legume (APL), diâmetro do coleto (DC), teor de nutrientes foliares, peso de mil grãos (PG), produtividade de grãos (PROD) e atributos químicos do solo.

Para a análise dos teores de nutrientes nas folhas, foram coletadas as amostras em fase de floração plena, sendo 10 trifólios com pecíolo em cada parcela para a determinação de macro e micronutrientes, conforme metodologia de Malavolta et al. (1997). As folhas foram submetidas à lavagem com água destilada. Após secagem em estufa de circulação forçada de ar a $65^{\circ} \mathrm{C}$ por 72 horas, posteriormente as folhas foram moídas em moinho tipo Wiley e peneiradas com peneiras de malha de 20 mesh $(0,85 \mathrm{~mm})$. O nitrogênio foi extraído por digestão sulfúrica a quente e determinado pelo método semi-micro Kjedahl. O $\mathrm{P}, \mathrm{K}, \mathrm{Ca}, \mathrm{Mg}, \mathrm{Cu}, \mathrm{Fe}, \mathrm{Mn}$ e $\mathrm{Zn}$ foram extraídos por digestão nítrico-perclórica a quente e determinados por espectrometria de absorção molecular (P), espectrofotometria de emissão de chama (K), espectrofotometria de absorção atômica $(\mathrm{Ca}, \mathrm{Mg}, \mathrm{Cu}$, $\mathrm{Fe}, \mathrm{Mn}$ e Zn).

Altura de planta: antes da colheita, a altura da planta de soja foi determinada a partir de cinco plantas, com régua graduada em centímetros, tomando-se a distância ente o nível do solo e o ápice da planta. Altura do primeiro legume: antes da colheita, medindo cinco plantas, com régua graduada em centímetros, com os valores representando a média entre as alturas de inserção do primeiro legume. Diâmetro de coleto: antes da colheita, o diâmetro do coleto da planta de soja foi determinada a partir de cinco plantas, com paquímetro em milímetros.

Peso de mil grãos: depois da colheita, homogeneizando os grãos colhidos a partir de plantas de cada parcela individualmente. Produtividade de grãos: foi determinada amostrando-se uma área de 4,5 $\mathrm{m}^{2}$, dentro de cada parcela. Após a trilha das plantas em trilhadora estacionária e limpeza dos grãos, as amostras foram colocadas em estufa de ventilação forçada e corrigida umidade para $13 \%$ e posteriormente foram pesadas em balança digital.

Após a colheita da soja, foram coletadas amostras de solo, nas camadas de 0-10 e 10-20 cm, para realizar as análises químicas de acordo com metodologia descrita por Claessen (1997), que englobaram: $\mathrm{pH}$ em água, $\mathrm{pH} \mathrm{CaCl}_{2}, \mathrm{Ca}, \mathrm{Mg}, \mathrm{Al}, \mathrm{P}$ Melich-1, K e MO.

\subsection{Análise estatística}

Os resultados obtidos, em cada variável analisada, foram submetidos à análise de variância, e as médias dos tratamentos comparadas pelo Teste t de Student a 5\%, utilizando o programa estatístico Sisvar (Ferreira, 2014). Os dados das doses de pó de rocha (significativos) foram submetidos também a análise de regressão. Os modelos para ajustes das equações foram escolhidos com base no coeficiente de determinação e na sua significância $(\mathrm{p}<0,01)$.

\section{Resultados e Discussão}

\subsection{Atributos químicos do solo}

\subsubsection{Camada superficial do solo $(0-10 \mathrm{~cm})$}

Não foi detectada interação entre o residual da aplicação das doses de pó de basalto e da adubação química complementar, somente efeito isolado da adubação química para as variáveis P e K (Tabela 2), onde os maiores teores de P e K no solo foram dos tratamentos que receberam adubação química, esses resultados que já eram esperados pela adição direta de $\mathrm{P}_{2} \mathrm{O}_{5}$ e $\mathrm{K}_{2} \mathrm{O}$, via adubação.

$\mathrm{Na}$ análise dos atributos químicos do solo na camada superficial $(0-10 \mathrm{~cm})$ percebe-se que os valores de $\mathrm{pH}, \mathrm{M} . \mathrm{O}, \mathrm{P}$, $\mathrm{Ca}, \mathrm{Mg}, \mathrm{Cu}, \mathrm{Mn}$ e $\mathrm{Zn}$ estão dentro dos níveis de suficiências de médios a altos teores para o desenvolvimento da cultura (Raij et al., 1997). 


\subsubsection{Camada sub superficial do solo $(10-20 \mathrm{~cm})$}

Observa-se que na camada subsuperficial os valores de pH, M.O, P, K, Ca, Mg, SB, CTC, V\%, Mn e Zn (Tabela 3) são menores que na camada de 0-10 cm (Tabela 2), esta é uma característica comum, visto que, é na camada superficial que os corretivos e adubos são adicionados. Já os teores de acidez potencial $(\mathrm{H}+\mathrm{Al})$ e Fe estão em maiores concentrações na camada de 10-20 cm, característica natural de solos do cerrado.

Não foi detectada interação entre o residual da aplicação das doses de pó de basalto e da adubação química complementar. Houve, somente efeito isolado da adubação química para a variável Mn (Tabela 3), onde o maior teor no solo foi encontrado nos tratamentos que não receberam a adubação química, possivelmente porque na camada subsuperficial o $\mathrm{pH}$ do solo encontra-se mais ácido, contribuindo com a maior solubilidade do Mn e consequentemente com sua disponibilidade (Carvalho, 2007).

$\mathrm{Na}$ análise dos atributos químicos do solo na camada subsuperficial $(10-20 \mathrm{~cm})$ observa-se que os valores de $\mathrm{pH}$, M.O, P, Ca, Mg, Cu, Mn e Zn estão dentro dos níveis médios a altos e se encontram suficientes para o desenvolvimento da cultura (Raij, et al., 1997).

\subsection{Análise foliar e produtividade}

Não foi detectada interação entre o residual da aplicação das doses de pó de basalto e da adubação química complementar, somente efeito isolado do residual da aplicação das doses de pó de basalto para as concentrações foliares de P, $\mathrm{Cu}$ e $\mathrm{Zn}$, que houveram diminuição nos teores foliares, possivelmente causado pelo efeito de diluição, devido ao maior acumulo de matéria seca nas folhas, e efeito isolado da adubação química complementar para a concentração foliar de Mg que foi superior nos tratamentos que não receberam a adubação química complementar (Tabela 4). Apesar da diferença significativa, os teores de Mg nas folhas se encontram na faixa adequada para o desenvolvimento da soja (Embrapa, 1996).

Para as variáveis $\mathrm{Cu}$ e $\mathrm{Zn}$ os dados se ajustaram a função quadrática (Figuras 1A e 1B). O menor teor de $\mathrm{Cu}$ encontrado nas folhas da soja foi de $10,90 \mathrm{mg} \mathrm{dm}^{-3}$ na dose de 6,71 $\mathrm{Mg} \mathrm{ha}^{-1}$ do pó de basalto (Figuras 1A). Para o Zn, o menor teor encontrado nas folhas da soja foi de $41,66 \mathrm{mg} \mathrm{dm}^{-3}$ na dose de 7,92 $\mathrm{Mg} \mathrm{ha}^{-1}$ do pó de basalto (Figura 1B). Apesar dos menores teores de $\mathrm{Cu}$ e $\mathrm{Zn}$ nas doses indicadas do pó de basalto, os teores estão na faixa adequada para o desenvolvimento das plantas de soja. Para o teor foliar de P os dados se ajustaram a função linear decrescente (Figura 1C). 


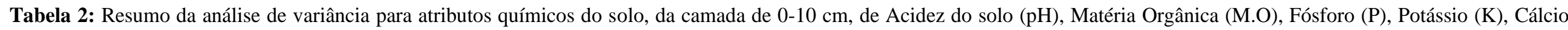

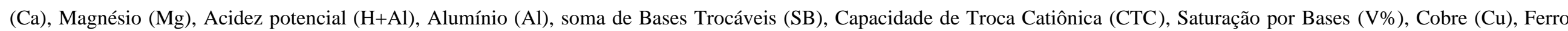
(Fe), Manganês (Mn) e Zinco (Zn) em relação a doses de pó de basalto e adubação química adicional, Dourados - MS, 2019.

\begin{tabular}{|c|c|c|c|c|c|c|c|c|c|c|c|c|c|c|c|}
\hline Tratamentos & $\begin{array}{c}\mathrm{pH} \\
\left(\mathrm{CaCl}_{2}\right)\end{array}$ & $\begin{array}{c}\mathrm{pH} \\
\left(\mathrm{H}_{2} \mathrm{O}\right)\end{array}$ & M.O & $\mathrm{P}$ & $\mathrm{K}$ & $\mathrm{Ca}$ & $\mathrm{Mg}$ & $\mathrm{H}+\mathrm{Al}$ & SB & CTC & $\mathrm{V} \%$ & $\mathrm{Cu}$ & $\mathrm{Fe}$ & $\mathrm{Mn}$ & $\mathrm{Zn}$ \\
\hline $\begin{array}{c}\text { Doses de pó de } \\
\text { basalto (D) } \\
\left(\mathrm{Mg} \mathrm{ha}^{-1}\right)\end{array}$ & & & $\mathrm{g} \mathrm{dm}^{-3}$ & $\mathrm{mg} \mathrm{dm^{-3 }}$ & ------ & -------- & --mmolcd & $\mathrm{Am}^{-3}----$ & ---o- & ---- & $\%$ & \multicolumn{4}{|c|}{------ $\mathrm{mg} \mathrm{dm}^{-3}$--- } \\
\hline 0 & 5,46 & 6,11 & 27,91 & 16,88 & 4,37 & 44,77 & 21,32 & 42,98 & 70,48 & 113,45 & 62,24 & 13,96 & 44,72 & 105,42 & 4,11 \\
\hline 2,5 & 5,45 & 6,11 & 27,84 & 18,14 & 4,00 & 43,78 & 21,49 & 44,25 & 69,29 & 113,51 & 61,08 & 14,17 & 42,79 & 107,26 & 3,25 \\
\hline 5,0 & 5,39 & 6,04 & 27,59 & 16,50 & 3,94 & 42,58 & 21,14 & 46,40 & 67,66 & 114,04 & 59,49 & 14,00 & 43,91 & 101,95 & 2,95 \\
\hline 7,5 & 5,50 & 6,12 & 27,20 & 15,66 & 3,92 & 42,90 & 21,82 & 44,51 & 68,64 & 113,12 & 60,61 & 14,22 & 45,20 & 105,35 & 3,09 \\
\hline 10,0 & 5,51 & 6,15 & 27,95 & 17,04 & 3,95 & 45,11 & 21,84 & 42,52 & 70,88 & 113,45 & 62,59 & 14,12 & 45,22 & 105,70 & 3,05 \\
\hline $\mathrm{Pr}>\mathrm{F}$ & $\mathrm{ns}$ & $\mathrm{ns}$ & ns & $\mathrm{ns}$ & $\mathrm{ns}$ & $\mathrm{ns}$ & $\mathrm{ns}$ & $\mathrm{ns}$ & $\mathrm{ns}$ & $\mathrm{ns}$ & $\mathrm{ns}$ & $\mathrm{ns}$ & $\mathrm{ns}$ & $\mathrm{ns}$ & $\mathrm{ns}$ \\
\hline \multicolumn{16}{|l|}{ Adubação (A) } \\
\hline Com & 5,44 & 6,08 & 28,06 & $19,3 \mathrm{a}$ & $4,33 \mathrm{a}$ & 44,21 & 21,57 & 44,65 & 70,11 & 114,77 & 61,13 & 14,11 & 43,36 & 105,60 & 3,19 \\
\hline sem & 5,48 & 6,13 & 27,33 & $14,4 \mathrm{~b}$ & $3,74 \mathrm{~b}$ & 43,45 & 21,47 & 43,61 & 68,66 & 112,26 & 61,26 & 14,08 & 45,37 & 104,67 & 3,38 \\
\hline $\operatorname{Pr}>\mathrm{F}$ & $\mathrm{ns}$ & ns & ns & $* *$ & $*$ & $\mathrm{~ns}$ & $\mathrm{~ns}$ & $\mathrm{~ns}$ & $\mathrm{~ns}$ & $\mathrm{~ns}$ & ns & ns & $\mathrm{ns}$ & $\mathrm{ns}$ & $\mathrm{ns}$ \\
\hline $\operatorname{Pr}>F(\mathrm{DxA})$ & ns & $\mathrm{ns}$ & ns & ns & $\mathrm{ns}$ & ns & ns & $\mathrm{ns}$ & ns & $\mathrm{ns}$ & ns & $\mathrm{ns}$ & ns & $\mathrm{ns}$ & ns \\
\hline $\mathrm{CV}(\%)$ & 4,89 & 3,60 & 5,87 & 14,79 & 19,83 & 9,18 & 9,50 & 16,40 & 8,60 & 4,13 & 9,15 & 4,33 & 14,50 & 6,56 & 33,88 \\
\hline Média & 5,46 & 6,11 & 27,70 & 16,84 & 4,04 & 43,83 & 21,52 & 44,13 & 69,39 & 113,51 & 61,20 & 14,10 & 44,37 & 105,14 & 3,29 \\
\hline
\end{tabular}

$\mathrm{ns}, * \mathrm{e}^{* *}$ : não significativo e significativo a $5 \%$ e $1 \%$ de probabilidade pelo teste de F, respectivamente. Fonte: Autores. 


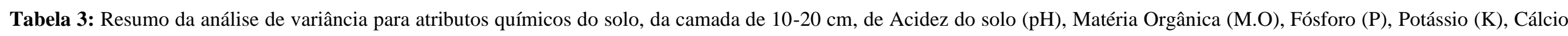

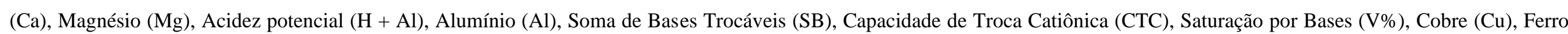
(Fe), Manganês (Mn) e Zinco (Zn) em relação a doses de pó de basalto e adubação química adicional, Dourados - MS, 2019.

\begin{tabular}{|c|c|c|c|c|c|c|c|c|c|c|c|c|c|c|c|}
\hline Tratamentos & $\begin{array}{c}\mathrm{pH} \\
\left(\mathrm{CaCl}_{2}\right.\end{array}$ & $\begin{array}{c}\mathrm{pH} \\
\left(\mathrm{H}_{2} \mathrm{O}\right)\end{array}$ & M.O & $\mathrm{P}$ & $\mathrm{K}$ & $\mathrm{Ca}$ & $\mathrm{Mg}$ & $\mathrm{H}+\mathrm{Al}$ & SB & CTC & $\mathrm{V} \%$ & $\mathrm{Cu}$ & $\mathrm{Fe}$ & $\mathrm{Mn}$ & $\mathrm{Zn}$ \\
\hline $\begin{array}{c}\text { Doses de pó de } \\
\text { basalto (D) } \\
\left(\mathrm{Mg} \mathrm{ha}^{-1}\right)\end{array}$ & & & $\mathrm{g} \mathrm{dm}^{-3}$ & $\mathrm{mg} \mathrm{dm}{ }^{-3}$ & ---- & 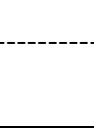 & $---m m o$ & $\mathrm{cdm}^{-3}--$ & & 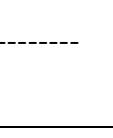 & $\%$ & \multicolumn{4}{|c|}{ 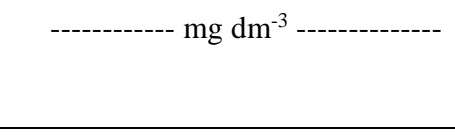 } \\
\hline 0 & 4,95 & 5,67 & 20,66 & 5,72 & 1,74 & 28,72 & 16,00 & 55,41 & 46,45 & 101,89 & 45,58 & 14,52 & 56,70 & 66,01 & 1,56 \\
\hline 2,5 & 5,05 & 5,74 & 21,19 & 5,3 & 1,62 & 27,91 & 16,09 & 53,16 & 45,62 & 98,80 & 46,24 & 14,10 & 53,99 & 65,82 & 1,54 \\
\hline 5,0 & 4,94 & 5,66 & 19,95 & 4,26 & 1,52 & 26,96 & 15,66 & 55,86 & 44,14 & 100,04 & 44,49 & 14,04 & 56,15 & 66,12 & 1,34 \\
\hline 7,5 & 5,04 & 5,77 & 19,95 & 5,19 & 1,39 & 27,99 & 16,67 & 54,89 & 46,04 & 100,91 & 46,00 & 14,29 & 55,77 & 68,90 & 1,34 \\
\hline 10,0 & 5,07 & 5,77 & 22,39 & 4,71 & 1,57 & 28,80 & 16,52 & 54,06 & 46,85 & 100,91 & 46,58 & 14,41 & 53,52 & 69,05 & 1,40 \\
\hline $\operatorname{Pr}>\mathrm{F}$ & $\mathrm{ns}$ & $\mathrm{ns}$ & ns & $\mathrm{ns}$ & $\mathrm{ns}$ & $\mathrm{ns}$ & $\mathrm{ns}$ & ns & ns & ns & ns & ns & $\mathrm{ns}$ & $\mathrm{ns}$ & $\mathrm{ns}$ \\
\hline \multicolumn{16}{|l|}{ Adubação (A) } \\
\hline Com & 4,96 & 5,69 & 20,26 & 5,37 & 1,64 & 28,51 & 15,79 & 56,24 & 45,06 & 101,32 & 44,49 & 14,20 & 55,24 & $63,34 b$ & 1,35 \\
\hline sem & 5,05 & 5,76 & 21,39 & 4,71 & 1,50 & 27,64 & 16,59 & 53,11 & 46,59 & 99,70 & 47,06 & 14,34 & 55,21 & $71,02 \mathrm{a}$ & 1,51 \\
\hline $\operatorname{Pr}>\mathrm{F}$ & $\mathrm{ns}$ & ns & $\mathrm{ns}$ & $\mathrm{ns}$ & $\mathrm{ns}$ & $\mathrm{ns}$ & $\mathrm{ns}$ & $\mathrm{ns}$ & $\mathrm{ns}$ & $\mathrm{ns}$ & ns & $\mathrm{ns}$ & $\mathrm{ns}$ & $*$ & $\mathrm{~ns}$ \\
\hline $\operatorname{Pr}>F(D x A)$ & $\mathrm{ns}$ & $\mathrm{ns}$ & $\mathrm{ns}$ & ns & $\mathrm{ns}$ & $\mathrm{ns}$ & ns & $\mathrm{ns}$ & $\mathrm{ns}$ & ns & $\mathrm{ns}$ & $\mathrm{ns}$ & $\mathrm{ns}$ & $\mathrm{ns}$ & $\mathrm{ns}$ \\
\hline $\mathrm{CV}(\%)$ & 4,37 & 3,06 & 9,11 & 37,75 & 33,25 & 12,71 & 10,27 & 14,83 & 11,15 & 6,69 & 12,37 & 4,10 & 11,76 & 8,44 & 20,28 \\
\hline Média & 5,01 & 5,72 & 20,83 & 5,04 & 1,57 & 28,08 & 16,19 & 54,68 & 45,82 & 100,51 & 45,77 & 14,27 & 55,23 & 67,18 & 1,43 \\
\hline
\end{tabular}

ns e *: não significativo e significativo a $5 \%$ de probabilidade pelo teste de $\mathrm{F}$, respectivamente. Fonte: Autores 
Research, Society and Development, v. 10, n. 6, e33710615599, 2021

(CC BY 4.0) | ISSN 2525-3409 | DOI: http://dx.doi.org/10.33448/rsd-v10i6.15599

Tabela 4. Teores foliares de macro e micronutrientes da soja, em função de doses de pó de basalto e da adubação química complementar.

\begin{tabular}{|c|c|c|c|c|c|c|c|c|c|}
\hline \multirow{3}{*}{$\begin{array}{c}\text { Tratamentos } \\
\begin{array}{c}\text { Doses de pó de } \\
\text { basalto (D) } \\
\left(\mathrm{Mg} \mathrm{ha}^{-1}\right)\end{array}\end{array}$} & \multicolumn{9}{|c|}{ Teores foliares de macro e micronutrientes } \\
\hline & $\mathrm{N}$ & $\mathrm{P}$ & $\mathrm{K}$ & $\mathrm{Ca}$ & $\mathrm{Mg}$ & $\mathrm{Cu}$ & $\mathrm{Fe}$ & $\mathrm{Mn}$ & $\mathrm{Zn}$ \\
\hline & \multicolumn{5}{|c|}{ 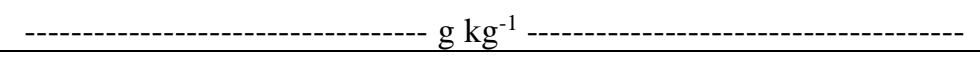 } & \multicolumn{4}{|c|}{ 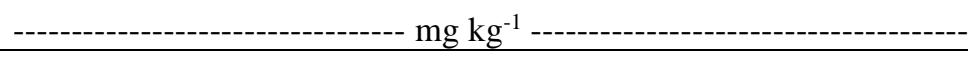 } \\
\hline \multicolumn{10}{|c|}{ Safra 2018/19 } \\
\hline 0 & 47,22 & 4,24 & 21,38 & 8,24 & 4,17 & 13,20 & 119,87 & 51,99 & 44,16 \\
\hline 2,5 & 45,25 & 4,18 & 21,81 & 8,30 & 4,12 & 11,81 & 108,24 & 50,02 & 42,82 \\
\hline 5,0 & 44,51 & 4,11 & 21,12 & 8,21 & 4,09 & 11,06 & 117,69 & 51,95 & 41,99 \\
\hline 7,5 & 43,79 & 4,04 & 21,69 & 8,39 & 4,06 & 10,95 & 108,45 & 49,77 & 41,69 \\
\hline 10,0 & 44,50 & 3,97 & 22,37 & 8,60 & 4,06 & 11,48 & 109,67 & 48,65 & 41,82 \\
\hline $\operatorname{Pr}>F(D)$ & ns & $* *$ & ns & $\mathrm{ns}$ & $\mathrm{ns}$ & $* *$ & ns & ns & $* *$ \\
\hline \multicolumn{10}{|l|}{ Adubação (A) } \\
\hline Com & 44,83 & 4,15 & 21,82 & 8,36 & $3,99 \mathrm{~b}$ & 11,25 & 115,51 & 49,78 & 40,88 \\
\hline Sem & 45,27 & 4,07 & 21,52 & 8,31 & $4,21 \mathrm{a}$ & 12,15 & 110,06 & 51,17 & 44,14 \\
\hline $\operatorname{Pr}>\mathrm{F}(\mathrm{A})$ & ns & $\mathrm{ns}$ & ns & $\mathrm{ns}$ & $* *$ & ns & $\mathrm{ns}$ & $\mathrm{ns}$ & ns \\
\hline $\operatorname{Pr}>\mathrm{F}(\mathrm{AxD})$ & $\mathrm{ns}$ & ns & ns & ns & $\mathrm{ns}$ & $\mathrm{ns}$ & $\mathrm{ns}$ & $\mathrm{ns}$ & ns \\
\hline $\mathrm{CV}(\%)$ & 5,78 & 3,77 & 6,85 & 4,94 & 3,82 & 12,77 & 17,02 & 17,44 & 6,68 \\
\hline Média & 45,06 & 4,11 & 21,67 & 8,35 & 4,10 & 11,70 & 112,78 & 50,48 & 42,51 \\
\hline
\end{tabular}

ns e **: não significativo e significativo a $1 \%$ de probabilidade pelo teste de $\mathrm{F}$, respectivamente. Fonte: Autores. 
Figura 1. Teor foliar de cobre (A), zinco (B) e fósforo (C) em plantas de soja (p<0,01) em função do residual das doses de pó de basalto
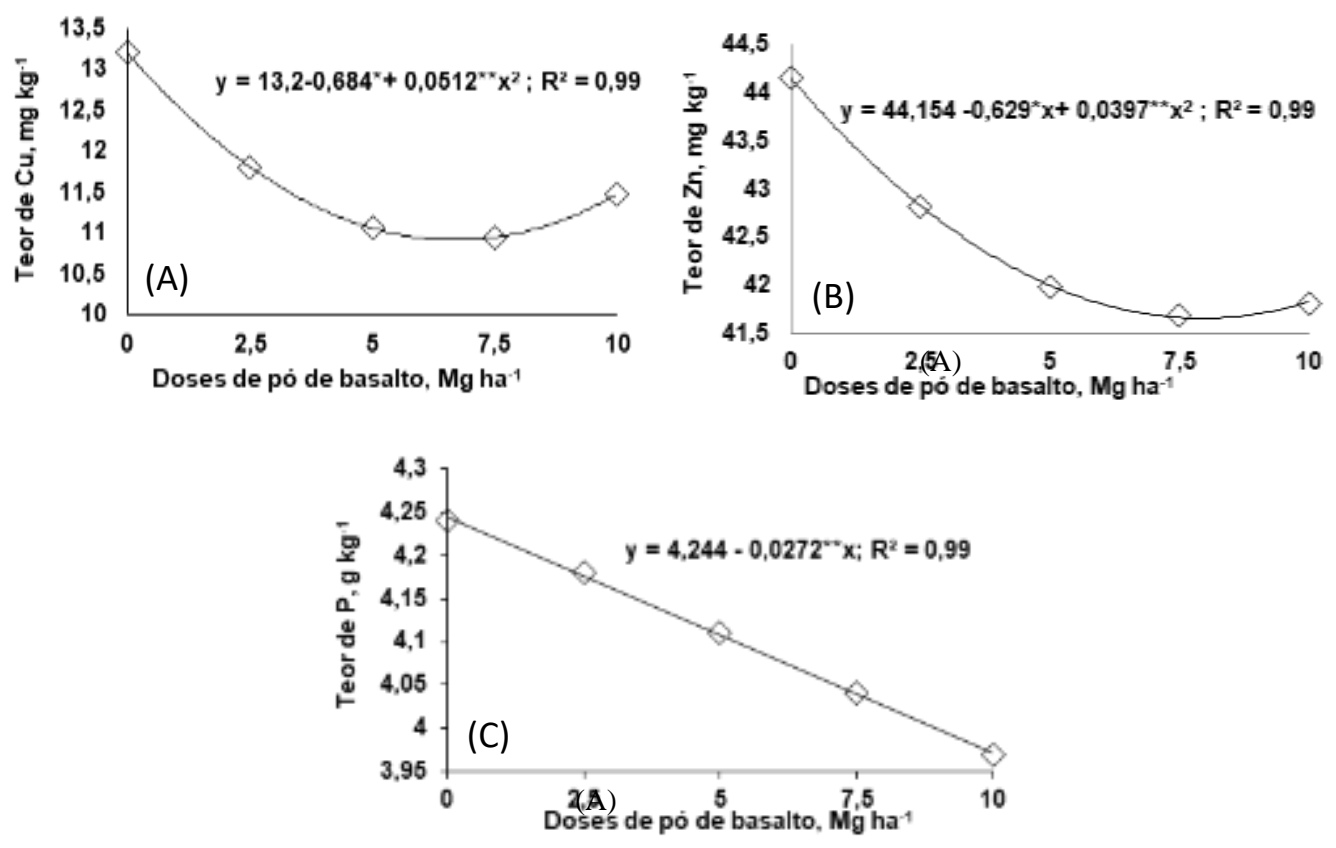

Fonte: Autores.

Considerando a análise foliar para a cultura da soja, somente o teor de $\mathrm{N}$ está abaixo do adequado, os demais nutrientes estão na faixa adequada para o desenvolvimento da cultura da soja (Broch \& Ranno, 2012), independente dos tratamentos.

Observando os resultados encontrados nos atributos químicos do solo (Tabelas 2 e 3) e foliar (Tabela 4), o solo da área experimental apresentava elevada reserva de nutrientes para as plantas, independente dos tratamentos. Solos com essa característica não respondem rapidamente à adubação e apresentam ótimos rendimentos das culturas (Klein, 2020).

Para os parâmetros avaliados quanto a biometria e produtividade não houve interação entre os fatores doses e adubação, somente houve significância $(\mathrm{p}<0,01)$ para o tratamento que recebeu adubação química, com os maiores valores encontrados nos tratamentos que receberam a adubação química complementar (Tabela 5).

A produtividade média deste experimento foi de $2.916,21 \mathrm{~kg} \mathrm{ha}^{-1}$, abaixo da média do Estado, que foi de $3.342 \mathrm{~kg}^{-1}$ (Famasul, 2020). Apesar do solo apresentar teores adequados de nutrientes para o desenvolvimento da cultura, a baixa produtividade encontrada no presente trabalho pode ser explicada pela fitotoxidez proporcionada pelo inseticida aplicado no estádio R5 da cultura.

Houve significância $(\mathrm{p}<0,01)$ para o peso de mil grãos somente utilizando a adubação química, onde os maiores valores foram encontrados nos tratamentos que receberam a adubação química complementar (Tabela 5).

O pó de basalto não influenciou as variáveis analisadas após dois anos da aplicação, o que indica que o material não pode ser utilizado como a principal fonte de nutrientes às plantas. Os dados apresentados referem-se aos principais resultados observados no segundo ano de análise do experimento com o cultivo da soja. Vale ressaltar que o experimento continua sendo amostrado e analisado periodicamente. Essa é a estratégia do estudo, para que se obtenham informações do efeito residual da aplicação do pó de basalto. 
Tabela 5. Altura final de plantas (ALT), altura da inserção do primeiro legume (APL), diâmetro do coleto (DC), peso de 1000 grãos (PG) e produtividade (Prod.) da soja, em função de doses de pó de basalto e da adubação química complementar.

\begin{tabular}{|c|c|c|c|c|c|}
\hline \multirow{2}{*}{$\begin{array}{c}\text { Tratamentos } \\
\text { Doses de pó } \\
\text { de basalto } \\
\text { (D) }\end{array}$} & \multirow[b]{2}{*}{ ALT } & \multicolumn{4}{|c|}{ Teores foliares de macro e micronutrientes } \\
\hline & & APL & $\mathrm{DC}$ & PG & Prod \\
\hline $\mathrm{Mg} \mathrm{ha}^{-1}$ & $\mathrm{~cm}$ & $\mathrm{~cm}$ & $\mathrm{~mm}$ & $\mathrm{~g}$ & $\mathrm{~kg} \mathrm{ha}^{-1}$ \\
\hline 0 & 86,76 & 22,77 & 7,00 & 83,55 & 2992,45 \\
\hline 2,5 & 85,81 & 21,94 & 7,46 & 83,86 & 2874,23 \\
\hline 5,0 & 84,60 & 22,04 & 7,00 & 83,30 & 3010,93 \\
\hline 7,5 & 83,86 & 21,34 & 7,71 & 85,31 & 2976,60 \\
\hline 10,0 & 82,49 & 23,06 & 7,32 & 85,02 & 2726,82 \\
\hline $\mathrm{Pr}>\mathrm{F}$ & $\mathrm{ns}$ & ns & Ns & $\mathrm{ns}$ & ns \\
\hline \multicolumn{6}{|l|}{$\begin{array}{l}\text { Adubação } \\
\text { (A) }\end{array}$} \\
\hline Com & $86,54 \mathrm{a}$ & 22,27 & 7,61 & $86,86 \mathrm{a}$ & $3148,87 \mathrm{a}$ \\
\hline Sem & $82,87 \mathrm{~b}$ & 22,18 & 6,98 & $81,56 \mathrm{~b}$ & $2683,54 \mathrm{~b}$ \\
\hline $\operatorname{Pr}>F$ & $*$ & $\mathrm{~ns}$ & $*$ & $* *$ & $* *$ \\
\hline $\begin{array}{l}\mathrm{Pr}>\mathrm{F} \\
(\mathrm{DxA})\end{array}$ & $\mathrm{ns}$ & ns & Ns & $\mathrm{ns}$ & ns \\
\hline $\mathrm{CV}(\%)$ & 5,46 & 20,25 & 10,41 & 3,92 & 14,45 \\
\hline Média & 84,70 & 22,23 & 0,73 & 84,21 & 2916,21 \\
\hline
\end{tabular}

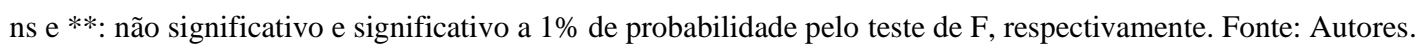

\section{Conclusão}

A adubação química influenciou os teores dos nutrientes $\mathrm{P}, \mathrm{K}$ e Mn no solo. Na camada de 0-10 cm houve aumento dos teores de P e K. Na camada de 10-20 cm ocasionou redução no teor de Mn.

O residual da aplicação das doses de pó de basalto ocasionou a redução das concentrações foliares de $\mathrm{P}, \mathrm{Cu}$ e $\mathrm{Zn}$ e, a adubação química complementar favoreceu a redução da concentração do Mg foliar, ambos possivelmente causado pelo efeito de diluição.

Altura de plantas, altura de inserção do primeiro legume, diâmetro do coleto, peso de mil grãos e produtividade foram influenciadas pela adição da adubação química complementar, com os maiores valores nos tratamentos que receberam a adubação química.

O pó de basalto não pode ser utilizado como a principal fonte de nutrientes às plantas.

Em função da baixa solubilidade do pó de basalto, recomenda-se que trabalhos com avaliações sucessivas dos atributos químicos do solo para avaliar a disponibilidade dos nutrientes a longo prazo.

\section{Referências}

Alovisi, A. M., Taques, M. M., Alovisi, A. A., Tokura, L. K., Silva, J. A. M. \& Cassol, C. J. (2020). Rochagem como alternativa sustentável para a fertilização de solo. Revista Gestão \& Sustentabilidade Ambiental, 9, 918-32.

Brasil. (2016). Instrução Normativa n 5, de 10 de março de 2016. Diário Oficial da União, República Federativa do Brasil, 10 e 11.

Broch, D. L. \& Ranno, S. K. (2012). Fertilidade do solo, Adubação e Nutrição da Cultura da Soja. Tecnologia de Produção: Soja e Milho 2012/2013. 2-38.

Carvalho, M. C. S. (2007). Nutrição e adubação do algodoeiro com micronutrientes. Embrapa. 
Research, Society and Development, v. 10, n. 6, e33710615599, 2021

(CC BY 4.0) | ISSN 2525-3409 | DOI: http://dx.doi.org/10.33448/rsd-v10i6.15599

Claessen, M. E. C., Barreto, W. O., Paula, J. L., \& Duarte, M. N. (1997). Manual de métodos de análise de solo. Embrapa.

Conab. (2020). Acompanhamento da safra brasileira de grãos, v.7- Safra 2019/20- Décimo levantamento. Conab.

Edward, W. O., Paula, A. M., \& Gatto, A. (2016). Influência do uso de pó de rochas fosfáticas e basálticas na ocorrência de micorrizas arbusculares em solo de cerrado. Brasília:UnB.

Embrapa. (1996). Centro Nacional de Pesquisa da Soja. Recomendações técnicas para a cultura da soja na Região Central do Brasil. Embrapa-Soja.

Famasul. (2020). Acompanhamento de safra. Soja 2019/2020. http://www.semagro.ms.gov.br/wp-content/uploads/2020/03/349-BOLETIM-SEMANAL-CASARURAL-AGRICULTURA-CIRCULAR-349.pdf.pdf.pdf.

Ferreira, D. F. (2014). Sisvar: um guia dos seus procedimentos de comparações múltiplas Bootstrap. Ciência e Agrotecnologia, 38 (2), 109-112. doi.org/10.1590/S1413-70542014000200001

Fietz, C. R., Fisch, G. F., Comunello, E., \& Flumignan, D. L. (2017). O clima da região de Dourados, MS. Embrapa Agropecuária Oeste.

Klein, Z. H. L. (2020). Alteração nos atributos químicos do solo após aplicação de pó de basalto como remineralizador.

Malavolta, E., Vitti, G. C., \& Oliveira, S. A. (1997). Avaliação do estado nutricional das plantas: princípios e aplicações. Piracicaba: Associação Brasileira de Potassa e do Fósforo.

Polidoro, J. C. (2019). Dependência externa de fertilizantes é debatido em audiência pública. Embrapa Notícias. https://www.embrapa.br/busca-de-noticias//noticia/30098853/dependencia-externa-de-fertilizantes-npk-e-debatida-em-audiencia-publica.

Raij, B. V., Cantarella, H., Quaggio, J. A., \& Furlani, A. M. C. (1997). Recomendações de adubação e calagem para o Estado de São Paulo. Campinas: IAC.

Santos, H.G., Jacomine, P. K. T., Anjos, L. H. C., Oliveira, V. A., Lumbreras, J. F., Coelho, M. R., Almeida, J. A., Filho, J. C. A., Oliveira, J. B., \& Cunha, T. J. F. (2013). Sistema brasileiro de classificação de solos. Embrapa Solos.

Schmidt, K. E., Cezimbra, J. C. G., Filho L. E. N. C., Bianchetto, R., Fontanive, D. E., \& Souza, E. L. (2019). Utilização do pó de rocha em substituição a adubação mineral tradicional na cultura da soja no noroeste do estado do RS. UERGS.

Tridge. (2019a). Top importing Countries of Nitrogen Fertilizers. https://www.tridge.com/intelligences/nitrogen-fertilizers/import.

Tridge. (2019b). Top importing Countries of Phosphate Fertilizers. https:/www.tridge.com/intelligences/phosphate-fertilizers/import.

Tridge. (2019c). Top importing Countries of Potassium Fertilizers. https://www.tridge.com/intelligences/potassium-fertilizers/import. 